\title{
Righting Writing's Wrongs: Toward Effective Writing Partnerships between Humans and AI
}

\author{
David Hyland-Wood ${ }^{1, *}$, Anna Harrison ${ }^{2}$, Ben Kolera ${ }^{3}$ \\ ${ }^{1}$ School of Information Technology and Electrical Engineering, The University of Queensland, St. Lucia, 4067 Queensland, Australia \\ ${ }^{2}$ The Cooler Moment, Brisbane, 4000 Queensland, Australia \\ ${ }^{3}$ Data 61, Brisbane, 4000 Queensland, Australia
}

Copyright (C) 2019 by authors, all rights reserved. Authors agree that this article remains permanently open access under the terms of the Creative Commons Attribution License 4.0 International License

\begin{abstract}
In industrialised nations, we take for granted that knowledge is acquired and shared through a written medium, and yet the actual task of writing remains a hard skill to master. In this paper, we present a blueprint for the integration of artificial intelligence (AI) into Web-based text editors, and show that this contributes towards simplifying the writing task for authors. Our approach utilises the textual content of a document as input into existing artificial intelligence processes, extended with an RDF knowledge graph. The supplementary material from the AI partner is presented to an author for possible inclusion in their document. We separately evaluated two approaches to the presentation of linguistic tone analysis into editors. Our results from unmoderated observational user tests validate the premise that cognitive technology can simplify the writing task, and show that design is critical to the adoption of cognitive technologies. We conclude with lessons learned from deploying informal qualitative research sessions and online observational tests, and share opportunities for future work. We note that merely applying AI or any new technology to a human activity is patently insufficient. Our limited experience and evaluation suggest that design and user testing are necessary activities for adoption and acceptance.
\end{abstract}

Keywords IT Education, Design, WYSWYG, AI, Cognitive Computing, Knowledge Graph, Linked Data

\section{Introduction}

In the short history of the World Wide Web, the rate at which new products, and entire product categories, have evolved has been staggering [8]. In the space of decades, the internet has delivered apps that have altered our patterns of behaviour, rendering us "addicted" to the worlds inside our mobile phones [16]. From shopping for food, to shopping for love, the way we do things now has changed.

While technological advances have altered many of our common daily activities, the user experience of What You See
Is What You Get (WYSIWYG) editing has remained surprisingly unchanged [13][11]. The process of content creation today is not that different to what it was in 1984: you click in a window, type some words, and click on $\mathbf{B}, I$ or $\underline{\mathrm{U}}$ buttons to make your writing more stylish. In fact, this process is so ubiquitous, and so ingrained in our daily habits that one could say that WYSIWYG has become like "water" [22]. From an innovation perspective, this is a dangerous (and boring) place to be.

Many types of writing, such as blog posts, academic or school assignments and business documents, could benefit from supplemental explanatory material to provide context, background, and additional information for exploration. The World Wide Web provides huge quantities of such supplementary material, but it is not generally suitable for inclusion in new writing due to issues of licensing, copyright, formatting, or syntax. Exceptions include clearly identifiable content meant to be embedded in hypertext documents, such as YouTube videos, and Twitter feeds.

At the point in time when a writer sits down to write, chances are they have already given some thought to what they will be writing about. Once they begin the process of writing, it is likely that they will iterate between the writing task and a number of auxiliary tasks, such as further research and concept development. In a traditional process, these auxiliary tasks will take place in a number of windows outside of (and independent of) the editor. Each interaction with a window outside of the text editor represents a context switch for the content writer, therefore adding to their cognitive load.

In this research, we explore the general hypothesis that the content creation process can be improved through the integration of auxiliary tasks directly into the editor environment. We wanted to determine whether the textual content in a general purpose hypertext editor may be used to automatically identify relevant supplementary material via AI processes, and whether that material may be presented to a document author for possible inclusion in a document in a useful manner.

The techniques necessary for such an exploration are now generally available. Linguistic parsing of text content may be conducted relatively easily using generally available tools, at 
least in many European languages including English. Concept identification from the results of linguistic parsing has been similarly successful.

Concepts may be matched to relevant additional information by querying a graph of information in the Resource Description Framework (RDF) data model [5]. Such information form "knowledge graphs" of related information.

The first general purpose knowledge graph of scale was Freebase [4], which became the basis for the Google Knowledge Graph [19]. Web search engines such as Google, Bing, and Yandex now use knowledge graphs to provide summaries of relevant information related to search terms. It is exactly this functionality that we wanted to add to our general purpose hypertext editors.

Following our research, Google announced a feature similar to the one described in this paper for their vertically-integrated Google Docs product [18]. The Google Docs "Explore" button opens a sidebar that exposes a search option, and presents concepts, images, and related information. Images may be dragged into a document; other presented content opens Web pages for further reading.

We separately explored the application of linguistic tone analysis to text editors to determine whether the many challenges faced by industry in applying tone analysis were due solely to the relative immaturity of the technology. We hypothesized that improved user interface designs could facilitate productive changes during the writing process. We created two user interface designs and tested them sequentially. The first design of a tone analysis user interface matched available information in a tone analyzer application programming interface (API). A subsequent design broke radically from available technical information to focus on information that could result in practical guidance. The initial design was evaluated using informal qualitative research sessions. The later design was evaluated using online unmoderated user tests.

\section{Materials and Methods}

\subsection{Architecture for alternative concepts}

We wrapped JavaScript-based hypertext Web editor, Text box.io, to include a sidebar for the presentation of supplemental material. The sidebar is an HTML component that accepts HTML fragments for display and possible drag-and-drop of components into the editor's content pane. JavaScript is used to query an online service to search for relevant information based upon the current contents of the editor.

Textual content is written into the editor by a document author. That content is uploaded to a cloud service for analysis (see Figure 1). The service sends the content to another service parsing, and to extract concepts. We used IBM's online AlchemyLanguage service ${ }^{1}$ for linguistic parsing and concept identification, although any number of similar services could have been used for those purposes. AlchemyLanguage is based upon IBM's Watson question-answering AI system [9]. One advantage of the AlchemyLanguage API is that its concept tagging

\footnotetext{
${ }^{1}$ https://www.alchemyapi.com/products/ alchemylanguage/
}

service $^{2}$ returns DBpedia [2], Yago [21] and Freebase URIs for each identified concept. We used DBpedia URIs as indexes into an RDF knowledge graph.

To implement our knowledge graph service, we used an Apache Tomcat Web application server with a RDF4J version 2.0 RDF database engine. Relevant portions of DBpedia were sharded into six RDF4J databases to speed both data indexing and assist query performance. DBpedia topical concepts, SKOS [12] categories, labels, image URLs, short abstracts, categories and their labels, transitive instance types, mappingbased objects and literals, and person data were used for our initial experiments. We limited our DBpedia extracts to the English language due to the language limitations of the AlchemyLanguage concept tagging service.

Two queries against the knowledge graph using the SPARQL 1.1 query language [10] were used to construct supplemental material representations:

- The first SPARQL query is used to identify the type of a concept (i.e. each concept will be either a person, place, organization, movie, species, product, or a general concept).

- The second SPARQL query is used to identify available data about a specific concept, as anticipated by its type.

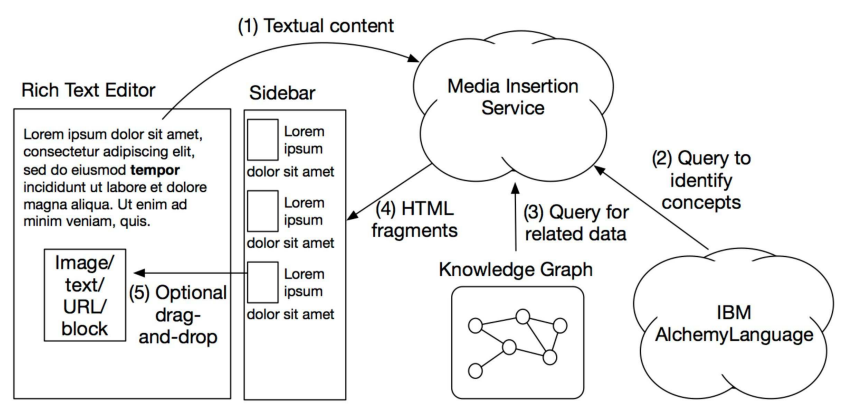

Figure 1. Data flow from editor to sidebar via enhancement services.

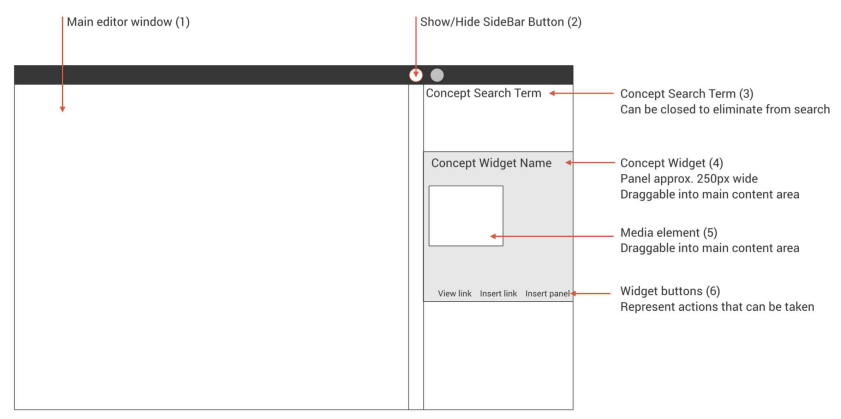

Figure 2. Editor and sidebar user interface elements

Thus, a concept of type "person" might have a birth date and a birth place. A place might have area and a population estimate, etc. Most data elements for any given type are considered to be optional. The results of the second query are used

\footnotetext{
${ }^{2}$ https://www.alchemyapi.com/products/ alchemylanguage/concepttagging
} 
to fill a type-specific HTML template. An HTML fragment is produced for each concept, and any empty divs are removed. The completed fragment is returned to the editor's sidebar for display.

SPARQL 1.1 SERVICE clauses were used to execute federated queries [17] across the various databases making up the knowledge graph.

We chose to construct our own knowledge graph instead of using one provided by the AlchemyLanguage service. We wish to eventually refine and contextualize the contents of the knowledge graph over time to provide industry- or even customerspecific data. We also wanted to be able to swap linguistic parsing and concept tagging services if desired at a later time.

A sidebar was added to our editors. Relevant components are illustrated in Figure 2. The show/hide button (2) opens and closes the sidebar panel, shrinking the main editor window content area (1) as it does so. The text typed into the main content area (1) is sent to the server for processing, and the top five concepts returned displayed as keywords (3) in the sidebar area. Individual concepts related to the search terms (3) are displayed as concept widgets (4). Each concept widget has a title and a combination of media and text items. Actions such as view and insert are displayed as buttons at the bottom of each concept widget (6). The concept widgets (4) and media items (5) are able to be drag and dropped into the main content area (1).

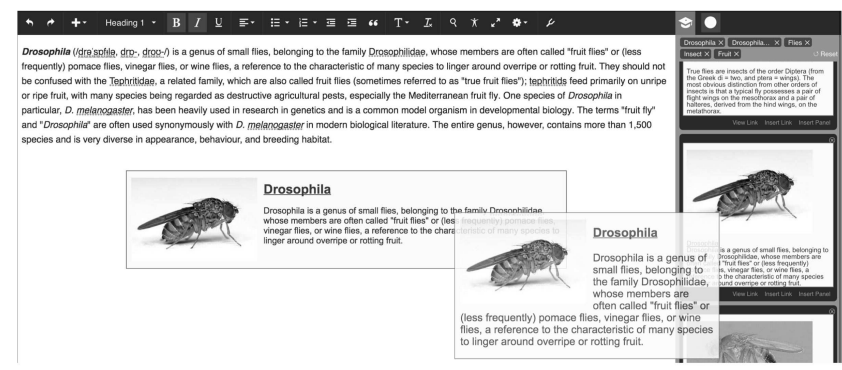

Figure 3. One possible interaction mode; drag-and-drop of suggested content. Content may be reformatted to match editor settings.
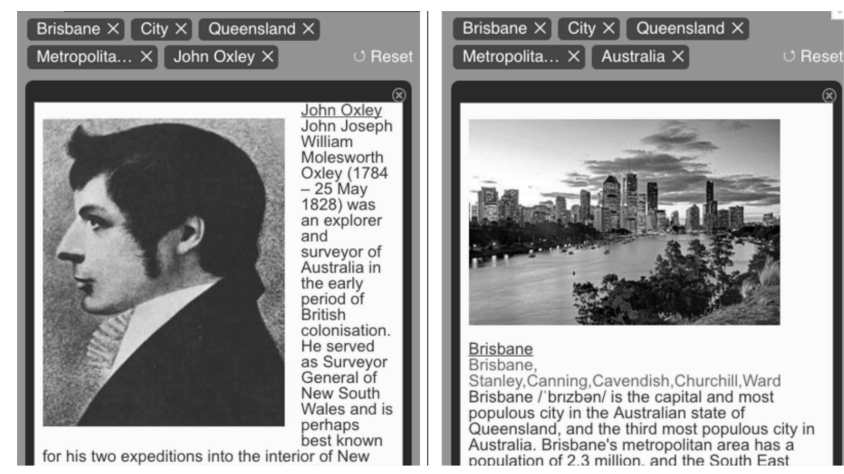

Figure 4. Concept filtering interaction sequence.

\subsection{Architecture for tone analysis}

we designed and implemented a textual tone analyzer UI component. The tone analyzer was integrated into two wi- dely used web-based WYSIWYG editors: TinyMCE and Textbox.io. The architecture of the textual tone analyzer component is shown in Figure 5.

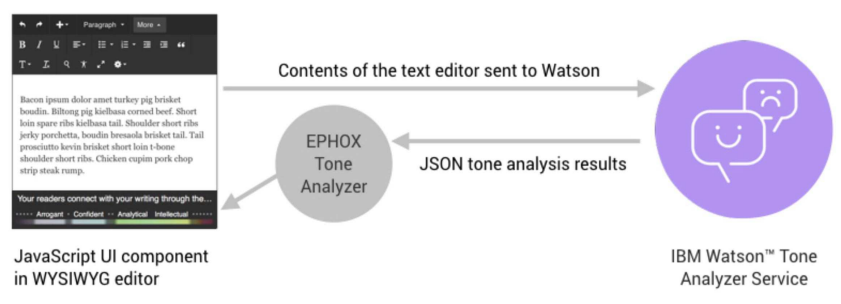

Figure 5. Architecture of the tone analyzer component.

The contents of the editor window are sent to the IBM Watson $^{\mathrm{TM}}$ Tone Analyzer Service ${ }^{3}$. Watson uses psycholinguistic analysis to process the input, and returns a file (in readily processable JSON format) containing the results of the analysis. The results are processed and represented as a UI component inside the text editor.

\subsection{Usage for alternative concepts}

We developed five interaction modalities to facilitate the adding of supplemental material related to alternative concepts to textual content in the editor:

1. Link insertion. Inserts a link to the knowledge graph concept.

2. Panel insertion. Inserts the linked knowledge graph concept widget as an in-line formatted blockquote.

3. Media insertion. Inserts the media (image or video) from the knowledge graph concept widget into the document.

4. Concept filtering. Removes concept search terms from the search query.

5. Link viewing. Opens the link to the knowledge graph concept in a separate browser window.

\subsubsection{Link insertion}

The first interaction type we implemented was the ability to simply add a link to the knowledge graph concept from the sidebar directly into the document. This functionality was delivered through an Insert link button on each concept widget. Pressing the Insert link button would insert a link at the current cursor position, or, if a selection was made in the editor the link would be applied to the selected text.

\subsubsection{Panel insertion}

The panel insertion interaction was implemented using two interaction styles. Firstly, like link insertion, a panel could be inserted into the document by clicking the Insert panel button on each concept widget. In addition, a drag-and-drop interaction was implemented on the panel, allowing the concept

\footnotetext{
${ }^{3}$ https://www.ibm.com/watson/developercloud/tone-analyzer.html
} 
widget to be dragged directly into the document. In both cases, the resulting concept was displayed as a formatted, in-line blockquote. The insert panel interaction sequence is shown in Figure 3.

\subsubsection{Media insertion}

The media insertion interaction was provided as a drag-anddrop interaction only, with no corresponding button on the concept widget. In this case, selecting a specific image (or video) from the sidebar and dragging it into the document embedded the media item only in-line in the document, the formatted blockquote was not included.

\subsubsection{Concept filtering}

Identified concepts were shown in a widget near the top of the sidebar (the "search terms" shown in Figure 3). The top five concepts identified by the AI service were listed as keywords within that widget. Each concept could be removed from consideration by deselecting its visual tag. Removal of a concept from the user interface removes it from the knowledge graph search. In the example shown in Figure 4, closing the "John Oxley" concept keyword results in that term being removed from the search, and all concept widgets related to that term being removed from the sidebar. The next most highly weighted concept is added to the widget if one is available.

\subsubsection{Link viewing}

The final interaction modality implemented allowed the viewing of the original knowledge graph concept in a new browser window. This was implemented as a View link button on each concept widget, and also as a hyperlink from the concept title. In both cases, clicking the button or the link opened the Wikipedia page related to the knowledge graph concept in a new browser tab.

\subsection{Usage for tone analysis}

Two designs of the tone analyzer component were created in order to evaluate a sub-hypothesis: Could output from existing AI systems be represented directly or would user experience design effort be necessary in order to match user expectations?

The initial design of the tone analyzer component presented all results obtained from Watson in a dialog-based UI component (Figure 6). The bar graph categories (Emotion Tone, Writing Tone and Social Tone) mirror the data architecture of the results returned from the Watson service.

The size of each bar graph corresponded to the numerical value assigned by Watson for each particular category of result. For example, in Figure 6, Watson reported an Emotional Tone of Anger with $12 \%$ confidence and a Social Tone of Openness with confidence of $67 \%$.

In our next iteration, we consciously made the decision to include an experienced UX designer in the development process from the start. We included the designer in the standard agile sprint process that was followed by the engineering team.

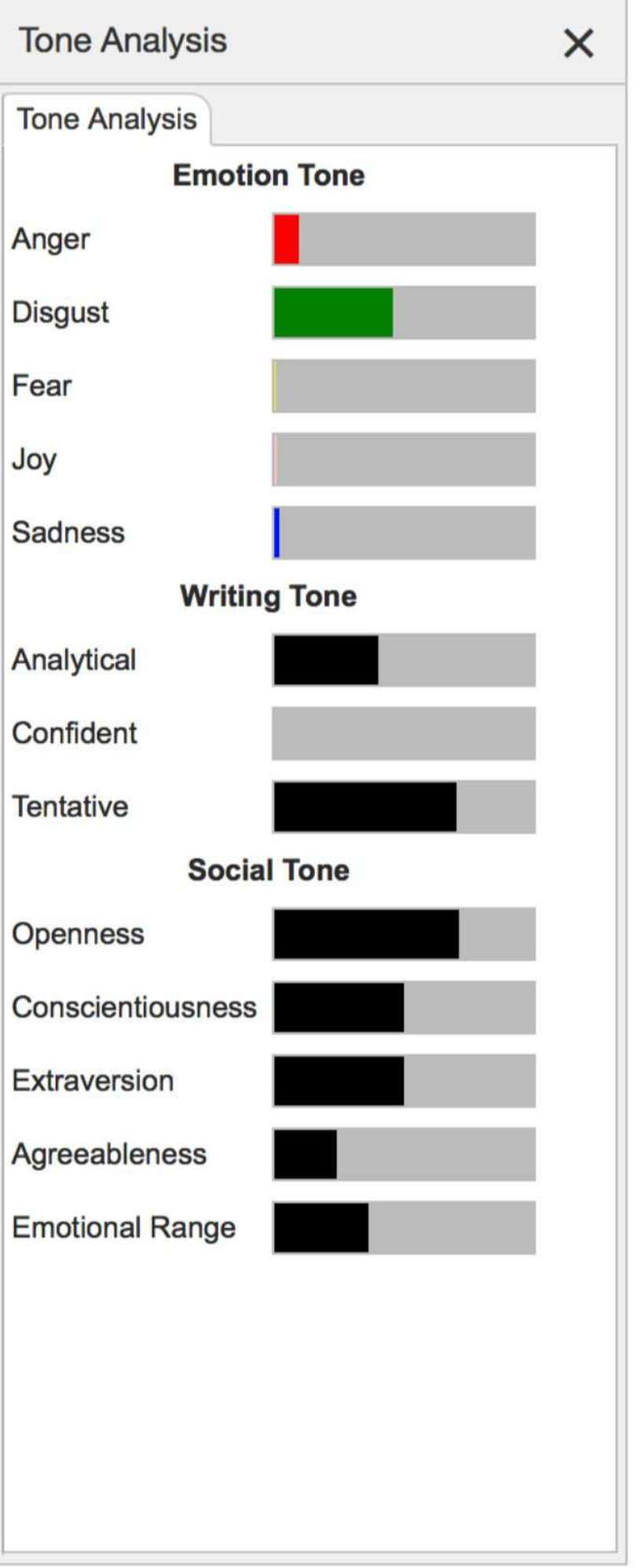

Figure 6. Initial tone analyzer design.

The redesign of the tone analyzer was founded on the two main insights gleaned from our initial user testing:

1. The data architecture of the results from Watson were not meaningful to a general content creation audience;

2. The tone analyzer needed to provide insights into what 
action could be taken to improve the reported writing tone.

In addition, we added a third requirement to reflect $\mathrm{UX}$ best practices to support editing on mobile platforms:

3. The tone analyzer should be supported on mobile and desktop platforms.

We did not anticipate the friction that resulted from the inclusion of design-led thinking into our traditional engineering process. Software developers used to working in the Scrum software development methodology had self-perceived shortterm deadlines due to deliverables being scheduled every two weeks. Design iteration naturally resulted in rework, much of which was initially resisted as being unwelcome. "Finished" components were often removed and even recreated leading to frustration from developers. Significant social efforts needed to be undertaken to resolve the issues.

We approached the redesign by making a decision to collapse the three distinct Watson categories into one. The intent was to remove or reduce the cognitive load associated with having to differentiate between three distinct classes of results.

Next, we addressed the presentation of the individual results within each category. We approached this step by analyzing the meaning of the results as reported in the Watson documentation $^{4}$. In the first design of the tone analyzer, we reported each result from Watson: For example, a Watson result of $49 \%$ for Openness was displayed as a corresponding bar graph.

In the redesign, we only showed results in which Watson had a high degree of confidence. For example, according to the Watson documentation, a result between $25 \%$ and $75 \%$ for Openness provides no reliable information about the writing tone. A score of less than $25 \%$ for Openness means that the writing tone is likely to be "no nonsense, straightforward or blunt"; while a score of more than $75 \%$ reflects a tone that is "intellectual, curious and emotionally aware".

We simplified these results further by associating a single word with each Watson result, and presenting the results in a binary format instead of a percentage. In the above example, a result of less than $25 \%$ was reported as "blunt" and a score of over $75 \%$ reported as "intellectual". No other results from the Openness category were reported in the UI component.

The decision to exclude all the information from the user interface highlighted a major difference in the approaches that are traditionally adopted by design and technology professionals. The notion that the user would not get all the information, or that the information presented was not completely accurate (we switched from reporting percentages to a binary on-off scale) horrified the developers and caught us by surprise. In hindsight, the severity of the friction on this point allowed us to address the issues in subsequent sprint cycles.

Examples of the redesigned tone analyzer component are shown in Figure 7 (desktop appearance) and Figure 8 (mobile appearance).

To address the lack of direction about what a content creator should do given a particular tone result, we created a mapping between each of the word results (such as blunt, or intellectual)

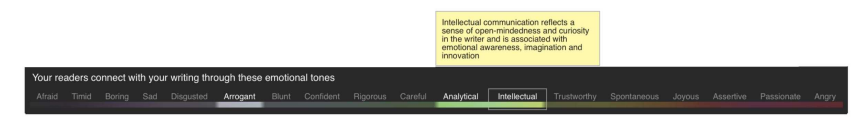

Figure 7. Redesigned tone analyzer, desktop appearance.

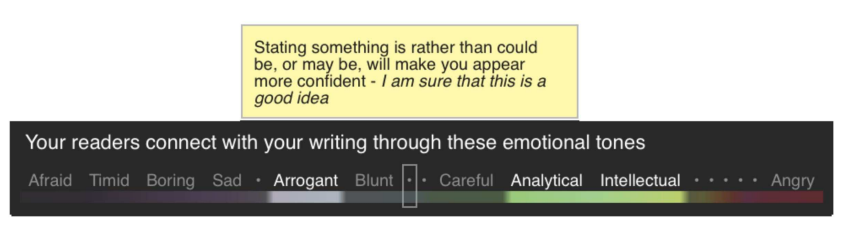

Figure 8. Redesigned tone analyzer, mobile appearance.

and the likely meaning of that tone being present in a piece of content. The actionable insights we developed were based on information in the Watson documentation, and supplemented with the authors' collective experience in linguistics.

For example, a blunt tone (openness $<=25 \%$ ) is likely to be interpreted as no-nonsense and straightforward, exhibiting little subtlety or complexity. An intellectual tone (openness $<=$ $75 \%$ ) reflects a sense of open-mindedness and curiosity in the writer and is associated with emotional awareness, imagination and innovation.

We also provided guidance on how to add a tone into a piece of content where a tone was not currently present. For example, to add more bluntness to one's writing, an author should stick to facts and simple instructions. Conversely, adding a little empathy can soften an otherwise blunt message. Making the tone more intellectual will communicate a balance between confident and analytical writing that is also engaging and interesting.

In order to create a UI component that was both intuitive and responsive, we looked into the literature on the links be- tween emotions and their representation as colors [14][7][3]. There are cultural differences in how people perceive color [15], however, as the Watson service is only available in English, we assumed a Western interpretation of mappings between color and emotion. In our example, we assigned the color aqua to "blunt" and green to "intelligent".

The word-actionable insight-color mappings were ordered to create a relative emotional spectrum. This allowed us to extrapolate colors for words for which the existing literature did not provide a color mapping. As an example, we extrapolated a grey-purple color for "timid" to reflect its position between "afraid" (dark-grey) and "boring" (purple). Table 1 shows the complete word-actionable insight-color mappings result set we developed for the Watson tone analyzer service.

The decision to represent the results as an emotion/color spectrum made the new design inherently responsive: as the emotional spectrum widget is resized from desktop (Figure 7) to mobile (Figure 8) sizes, the words representing the results are sequentially converted into dots. Meaning of the tone results is preserved through the color representation.

\footnotetext{
${ }^{4}$ https://www.ibm.com/watson/developercloud/doc/toneanalyzer/understanding-tone.shtml
} 
Table 1. IBM Watson Tone Analyzer Service Results mapped to Meaning-Color-Action triples

\begin{tabular}{|c|c|c|c|}
\hline Watson Result & Meaning & Color & Action \\
\hline $\begin{array}{l}\text { Emotional Tone: } \\
\text { Fear }>75 \%\end{array}$ & Afraid & & $\begin{array}{l}\text { Acknowledging fear can help to open a conversa- } \\
\text { tion around problem resolution - I am concerned } \\
\text { that we will go over budget; I am scared that our } \\
\text { product will ship late. }\end{array}$ \\
\hline $\begin{array}{l}\text { Language Tone: } \\
\text { Tentative }>75 \%\end{array}$ & Timid & & $\begin{array}{l}\text { Infusing more of a timid or tentative tone can help } \\
\text { to diffuse a potentially emotive or explosive situa- } \\
\text { tion - Would it be possible...? }\end{array}$ \\
\hline $\begin{array}{l}\text { Social Tone: } \\
\text { Extraversion }<25 \%\end{array}$ & Boring & & $\begin{array}{l}\text { Removing emotive or descriptive words can make } \\
\text { the tone more boring, an outcome that is desirable } \\
\text { in certain communications - The getaway vehicle } \\
\text { was a blue Holden. }\end{array}$ \\
\hline $\begin{array}{l}\text { Emotional Tone: } \\
\text { Sadness }>75 \%\end{array}$ & Sad & & $\begin{array}{l}\text { Acknowledging sadness can invoke sympathy, even } \\
\text { in the face of bad news - I am sorry that I missed } \\
\text { your presentation. }\end{array}$ \\
\hline $\begin{array}{l}\text { Emotional Tone: } \\
\text { Disgust }>75 \%\end{array}$ & Disgusted & & $\begin{array}{l}\text { Disgust is a normal reaction to exceptional or ex- } \\
\text { treme negative situations. Acknowledging feelings } \\
\text { of disgust can help to catalyze resolution of the ne- } \\
\text { gative situation. }\end{array}$ \\
\hline $\begin{array}{l}\text { Social Tone: } \\
\text { Agreeableness }<25 \%\end{array}$ & Arrogant & & $\begin{array}{l}\text { At times, it may be appropriate to focus commu- } \\
\text { nication around oneself, however, an arrogant tone } \\
\text { does this with a lack of compassion for others. Aim } \\
\text { for confidence or assertiveness instead. }\end{array}$ \\
\hline $\begin{array}{l}\text { Social Tone: } \\
\text { Openness }<25 \%\end{array}$ & Blunt & & $\begin{array}{l}\text { Blunt communication can be useful when dealing } \\
\text { with situations that can be misinterpreted. Sticking } \\
\text { to facts and simple instructions can help to reduce } \\
\text { complexity and subtlety. }\end{array}$ \\
\hline $\begin{array}{l}\text { Language Tone: } \\
\text { Confidence }>75 \%\end{array}$ & Confident & & $\begin{array}{l}\text { Stating something is rather than could be, or may } \\
\text { be, will increase the confidence in your communi- } \\
\text { cation. }\end{array}$ \\
\hline $\begin{array}{l}\text { Social Tone: } \\
\text { Conscientiousness }>75 \%\end{array}$ & Rigorous & & $\begin{array}{l}\text { Introducing a systematic writing plan can improve } \\
\text { the rigour of your communication. }\end{array}$ \\
\hline $\begin{array}{l}\text { Social Tone: } \\
\text { Emotional } \\
\text { Range }<25 \%\end{array}$ & Careful & & $\begin{array}{l}\text { A calm tone helps to diffuse difficult situations and } \\
\text { is useful for sensitive communications. Introducing } \\
\text { some emotional words will keep the writing from } \\
\text { being too bland. }\end{array}$ \\
\hline $\begin{array}{l}\text { Language Tone: } \\
\text { Analytic }>75 \%\end{array}$ & Analytical & & $\begin{array}{l}\text { Analytical writing is most valued in scientific and } \\
\text { business communications. If writing on more per- } \\
\text { sonal topics, it will help to introduce some emotio- } \\
\text { nal words to improve engagement. }\end{array}$ \\
\hline $\begin{array}{l}\text { Social Tone: } \\
\text { Openness }>75 \%\end{array}$ & Intellectual & & $\begin{array}{l}\text { An intellectual tone represents a fine balance bet- } \\
\text { ween confident and analytical writing that is also } \\
\text { engaging and interesting. Adding anecdotes and ex- } \\
\text { amples can help to shift the tone from analytical to } \\
\text { intellectual. }\end{array}$ \\
\hline $\begin{array}{l}\text { Social Tone: } \\
\text { Agreeableness }>75 \%\end{array}$ & Trustworthy & & $\begin{array}{l}\text { Gaining trust in communication can be more po- } \\
\text { werful than focusing on facts alone. Showing that } \\
\text { one cares and sympathizes with a situation fosters } \\
\text { trustworthiness. }\end{array}$ \\
\hline $\begin{array}{l}\text { Social Tone: } \\
\text { Conscientiousness }<25 \%\end{array}$ & Spontaneous & & $\begin{array}{l}\text { Spontaneity and lack of convention can be powerful } \\
\text { if used sparingly to surprise the reader. In general } \\
\text { communication, a more organized and systematic } \\
\text { approach will help to guide the reader to a desired } \\
\text { conclusion. }\end{array}$ \\
\hline
\end{tabular}




\begin{tabular}{|l|l|l|l|}
\hline $\begin{array}{l}\text { Emotional Tone: } \\
\text { Joy }>75 \%\end{array}$ & Joyous & $\begin{array}{l}\text { Joy is a natural response to positive situations. It } \\
\text { can be powerful when used to show gratitude, or } \\
\text { congratulate a colleague on a job well done. }\end{array}$ \\
\hline $\begin{array}{l}\text { Social Tone: } \\
\text { Extraversion }>75 \%\end{array}$ & Assertive & $\begin{array}{l}\text { Used sparingly, an assertive tone can inspire and } \\
\text { engage. To balance being assertive without beco- } \\
\text { ming arrogant, maintain a sense of compassion to- } \\
\text { wards others in your writing. }\end{array}$ \\
\hline $\begin{array}{l}\text { Social Tone: } \\
\text { Emotional } \\
\text { Range }>75 \%\end{array}$ & Passionate & $\begin{array}{l}\text { A passionate tone lights up emotive responses in ot- } \\
\text { hers. It can be used as a catalyst for action, as long } \\
\text { as it is tempered with sensitivity to one's surroun- } \\
\text { dings. }\end{array}$ \\
\hline $\begin{array}{l}\text { Emotional Tone: } \\
\text { Anger }>75 \%\end{array}$ & Angry & $\begin{array}{l}\text { Although anger is a normal reaction to injustice and } \\
\text { conflict in the world, it can sometimes be used to } \\
\text { mask feelings of insecurity. Examine reasons for } \\
\text { your anger, and consider expressing your emotions } \\
\text { in a more careful and analytical way. }\end{array}$ \\
\hline
\end{tabular}




\section{Evaluation}

\subsection{Evaluating the Cognitive Assistant}

The usage model for the Cognitive Assistant was evaluated via an unmoderated observational research study. Participants were given a writing task and asked to enrich their writing making use of the Cognitive Assistant. The task description given to users was:

Your Task: Imagine you are writing a blog post to tell your friends about a holiday you recently took (or would like to take) to New York City. Write your story in the editor window below.

Using the Cognitive Assistant, enhance your story with:

- Information about the city itself

- Information about any attractions, landmarks, shows, movies or restaurants that you visited (or would like to visit).

- Information about famous sports teams based in New York City

Please include at least one quote and one video.

The test task intentionally did not prompt the participants on how to achieve the task as we were interested in exploring the natural way in which participants would interact with the sidebar element. Participants were instructed to use a "talk aloud" protocol while completing the task and their interactions recorded using the online testing service Validately ${ }^{5}$.

The test was deployed to 20 participants aged over 18 years old and having some university education. Of the 20 participants, 11 were female and 9 were male. Thirteen tests were completed either in whole or in part.

\subsection{Evaluating the "raw" tone analysis presentation}

The user interface presenting "raw" results from the tone analyzer was implemented and showcased to commercial enterprise customers in early 2016 with the goal of collecting preliminary feedback to assess the feasibility of the project and the effectiveness of the design. Results were presented exactly as they were returned by the API with all information represented.

Informal, semi-structured interviews were conducted with 6 customers in Australia, Europe and the USA during a number of product-preview sessions. A debriefing session was held with the interviewer and authors in March.

Analysis of the interview notes suggested that clients were receptive to the idea of an $\mathrm{AI}$ enriched editor:

I can see the potential for a feature such as this one to help us out with writing...

However, it was immediately apparent that the presentation of all information was overwhelming to many users. The simplistic presentation of tone information severely lacked utility:
... but before I can use this tone analyser, I would have to do some training to understand what all these graphs mean.

This early feedback prompted us to conduct further in-house user tests. In April 2016 we recruited 5 bloggers from a local WordPress meetup, and conducted informal observational research sessions.

The participants were shown the tone analyzer and asked to describe how they might use it when writing a blog post. Our key goal was to articulate a realistic use case for the feature, and identify specific obstacles to usability in the early design. The sessions were audio-recorded.

The results confirmed that while participants were initially excited about the idea of an "assistant" in the editor, they did not know how to use our tone analyzer:

I mean... what does it mean if my Social Tone is $12 \%$ open? Should I be worried? How can I fix it?

I really am not sure what these categories [Emotional, Writing, Social] mean.

Evaluation of the "raw" tone analysis information could not effectively proceed because the information returned from the Watson service were not meaningful to a general audience. Almost all of the 11 participants directly expressed confusion around the categorization of the results (Emotion, Writing, Social), and of the meaning of the bar graphs within each category.

\subsection{Evaluating the designed tone analysis presentation}

We tested the re-designed tone analyzer by running an unmoderated observational research study deployed on-line via the online testing service Validately ${ }^{6}$. Participants were given a writing task and asked to enrich their writing using the tone analyzer:

Your Task: Imagine you are writing a blog post to tell your friends about a holiday you recently took (or would like to take) to New York City. Write your story in the editor window below.

Include:

- Information about the city itself

- Information about any attractions, landmarks, shows, movies or restaurants that you visited (or would like to visit)

- Information about famous sports teams based in New York City

Check your writing tone using the tone analyzer and make any adjustments if needed.

The instructions intentionally did not prompt the participants on how to achieve the task as we were interested in exploring the natural way in which participants would interact with the 
tone analyzer. Participants were instructed to use a "talk aloud" protocol while completing the task and their interactions recorded via the Validately service.

The test was deployed to 20 participants aged over 18 and having some university education. Of the 20 participants, 11 were female and 9 were male. Nine tests were completed in full.

\subsection{Observations regarding online test deployment}

The observational research for this study was conducted online using the Validately service. This style of unmoderated, remote user testing is fairly new to us, and revealed a number of insights that may be relevant to others considering this medium for user testing.

Overall, on-line user testing provides fast and cheap access to participants ${ }^{7}$. For example, the Validately service charges $\$ 107$ per unmoderated user test with a turnaround time of less than 24 hours. The accessibility of this type of testing service opens the opportunity to conduct more frequent user testing.

Unfortunately, unmoderated on-line tests are much less forgiving of test design imperfections than in-person tests. As the tests are deployed remotely, there is no ability to make micro adjustments during the course of the test - for example, in the tests we conducted for this paper, only 9 out of 20 participants completed the task. The main causes for test incompletion were that participants could not see the tone analyzer, either due to:

1. The tone analyzer button being hidden by a Validately show/hide task button; or

2. The tone analyzer opening up out of view in the Validately recorder window.

In both of the above cases, if the test was being conducted in person, the participant could have been alerted of these conditions without impacting the results of the study.

We also noted an interesting discrepancy in results between participants' actions and words, and the responses they provided to a question asked at the completion of the task. After the test, participants were asked to rate how useful they found the tone analyzer on a scale of 0 (not at all useful) to 10 (super useful). The average rating returned by participants who did not complete the test was 2.3 , with three participants scoring 5 and 7 for a feature they did not even engage with!

This result may reflect an underlying pressure that on-line participants feel to "do well" on the test as they are being compensated for it. As the field of remote user-testing grows, this aspect could warrant its own track of investigation.

\section{Results}

\subsection{Evaluating the Cognitive Assistant}

Videos were manually coded against the five-part usage model described in section 3, namely: (1) link insertion; (2) panel

\footnotetext{
${ }^{7}$ Cost per unmoderated user test as at October 2016, exclusive of monthly subscription fees.
}

insertion; (3) media insertion; (4) concept filtering and (5) link viewing. For each of these categories, two of the authors independently evaluated whether the participant:

\section{Found the interaction modality (DISCOVERABLE) \\ 2. Was able to interact in an intuitive way (INTUITIVE) \\ 3. Perceived the interaction as having value (USEFUL)}

The results were triangulated and discrepancies resolved by reviewing the video footage ${ }^{8}$. Results are shown in Table 2.

\subsubsection{All interactions}

Most participants intuited what the sidebar represented. Some representative comments included:

Looking at this cognitive assistant, that is interesting. The internet tells me a lot, about what New York is about, and I can insert it right here. It gives me a lot of extra information that I need, and it makes writing very simple because it's integrated.

...this is a great concept! I love the idea of a search engine running in the background, finding information to strengthen my writing.

This readily helps me to create a blog as it makes it very easy and quick to write. . . and it guides me with information that inspires me to write more, so I don't have writers block. It's very intuitive, and helps me to write a story with an abundance of information for the viewers of my blog.

[opens cognitive assistant] Oh, how interesting! So it looks like, maybe it searched the text [in the document] and used a search engine to look up the information from my text. That's very interesting.

12 of the 13 participants who completed the task were able to interact with the sidebar to varying degrees of success: the majority of interaction modalities were engaged with $(54 \%)$, over a third $(39 \%)$ of the interactions appeared intuitive and around a third (32\%) appeared useful.

A few participants commented on the sidebar being a source of inspiration for ideas that they had not thought of themselves.

The New York Giants... actually I don't follow sports so I will use the cognitive enhancer [to get some info on this topic].

[on opening cognitive assistant] Oh that's cool. It shows me facts about the Statue of Liberty... I like this system, how you can get facts on what you are typing about.

\footnotetext{
${ }^{8}$ An 8-minute summary video of the most useful results is available at https://w3id.org/people/prototypo/papers/WWW2017bvideo
} 
Table 2. Results from user tests

\begin{tabular}{|c|c|c|c|}
\hline & Discoverable & Intuitive & Useful \\
\hline All interactions (1, 2, 3, 4 5), $\mathrm{n}=35$ & $54 \%$ & $39 \%$ & $32 \%$ \\
Any insert interaction (1 or 2 or 3), $\mathrm{n}=13$ & $100 \%$ & $92 \%$ & $85 \%$ \\
Concept filtering interaction (4), $\mathrm{n}=11$ & $85 \%$ & $31 \%$ & $8 \%$ \\
Link viewing interaction (5), $\mathrm{n}=2$ & $16 \%$ & $16 \%$ & $16 \%$ \\
\hline
\end{tabular}

\subsubsection{Any insert interactions}

The three interaction modalities (link, panel and media insertion) were implemented as alternate modes of interaction. Looking at the results, all of the participants (100\%) figured out some way to interact with the sidebar in an intuitive way (92\%), and most found these interactions useful (85\%).

Link insertion was the least engaged with mode of interaction: only 3 of the 13 participants directly inserted a link using the Insert link button on the concept widget. 9 out of the 13 participants intuitively inserted a panel from the sidebar into their document (using either drag and drop or pressing the Insert panel link). Media insertion was the most intuitive interaction mode, with 10 out of 13 participants dragging media from the sidebar into the document. This result suggests that incorporating images and videos into the concept widgets in the sidebar will promote interaction (as opposed to text-only concept widgets).

For some, while analysis of their interactions revealed that they interacted with the sidebar as expected, their words provided a different narrative:

[uses the cognitive assistant to successfully insert a video and a concept panel] I'm going to say that because it was a prototype, I was unable to use the cognitive assistant.

The above result is not uncommon in this type of testing, and underscores the benefits of observational research over surveystyle methods: i.e. the truth lies in what participants do, not what they say that they do.

\subsubsection{Concept filtering}

The concept filtering interaction modality was discovered by $85 \%$ of participants, however, it was also found to be very confusing: less than a third of participants $(31 \%)$ found the feature intuitive, and only $8 \%$ appeared to find it useful.

Analysis of the participant interactions shows that the main cause for the drop in intuitiveness and utility related to the inability to perform a custom search and to the relevance of the results, i.e. what was returned from the AI service itself. Almost all participants expressed a desire to augment the concepts presented with a custom search feature:

When it came to putting the links in, they just did not have what I was looking for... they showed things that were not as [relevant] to me.

The results [in the sidebar] are not that relevant... I wish I could control these keywords, like if there was a search bar up here and I could search for New York Giants. I don't see a way to do that. The search results don't really support what I'm writing about.

Is there a way to search this? Because different things pop up here. . . if I was were planning a trip then I could say Oh Ellis Island, Oh yeah, I forgot about that and then I could go there [on concept filters] I don't understand what these things are for, I keep xing these thing out and different ones keep popping up...is it figuring out different things based on what I am writing?... I don't see a place to search for exactly what I want.

I like the idea of having the cognitive assistant right in the page where the typing is. It didn't seem to be easy to figure out what the search terms were.

The cognitive assistant does have a lot more potential, the only thing is they need to work on technical issues as it's hard to manage [the concept widgets] and search and sometimes you want it to find something, but it does not find it.

\subsubsection{Link viewing}

Only 2 of the participants (16\%) viewed, or expressed a desire to view, the original sources that corresponded to each concept widget in the sidebar. This result suggests that information presented in the sidebar innately took on a perceived level of authority that one would associate with an encyclopedic-type reference source - the links we tested were all pointing to $\mathrm{Wi}$ kipedia.com, but need not have been as the architecture of the sidebar allows for the integration of any data source. Other links in our products point to content from additional reachable sources (e.g. content available within an IBM Connections deployment). More research is warranted, but based on our results, care should be taken to ensure that a sidebar type integration into an editor presents reliable information to the author.

\subsubsection{Observations on the on-line testing method}

This research used traditional qualitative observational style research methods [6], carried out in a nontraditional way using the Validately service. Although many of the principles of observational research apply to the design and analysis of the user tests, we discovered interesting nuances in the data collection phase.

Firstly, although the task was relatively simple, our pre-tests indicated that a general audience was unable to complete the writing task. We adjusted the demographics to filter the sample 
population to include only those participants who had some university-level education. Even then, only $65 \%$ of participants were able to complete the test in part or in full.

Secondly, although the participants had some university level education, the task itself was perceived as very hard. $35 \%$ of the participants abandoned the test as they were unable to complete the writing component of the task:

I could not complete this task as I have never been to New York and so did not know what to write about.

At the completion of the user test, participants were asked to rate how useful they found the cognitive assistant on a scale of 0 (not at all useful) to 10 (extremely useful). We observed very little correlation between the rating participants assigned and how they interacted with the tool in the test. For example, the average rating for participants who did not even open the sidebar was 2.4 , with two of the respondents rating a 6 ! This result may reflect an underlying pressure that on-line participants feel to "do well" on the test as they are being compensated for it [1]. As the field of remote user testing grows, this aspect could warrant its own track of investigation.

\subsection{Results from the "raw" tone analysis presentation}

The results from our preliminary user tests indicated that:

1. The concept of a tone analyzer component integrated into the text editor was perceived as interesting to our participants;

2. The first version of the tone analyzer, which mirrored the underlying technology in the user interface of the component, resulted in no perceived utility to the participants.

It was immediately clear that the presentation of too much "technical" information was overwhelming and not useful.

Analysis of the comments made by participants during the observational research sessions suggested that the data architecture of the Watson results was not very meaningful to people without training in linguistics. Most of the participants echoed the sentiments quoted above, i.e. they did not understand fully what the categories (Emotion, Writing, Social) meant, nor what actions they should take as a result to improve their writing. These insights were key inputs to how we approached the re-design of the tone analyzer component.

We were going to need to present information in a manner easier for users to understand if tone analysis were to become a effective tool for writing. We (re)learned the importance of putting a human face on technology to create effective partnerships.

\subsection{Results from the designed tone analysis presentation}

The results from the 9 completed tests were evaluated independently by two of the authors. The video footage ${ }^{9}$ was coded against three criteria:

\footnotetext{
${ }^{9}$ An 8-minute summary video of the most useful results is available at https://w3id.org/people/prototypo/papers/WWW2017bvideo
}

1. ENGAGEMENT. The participant engaged with the tone analyzer $(=1)$. The participant did not engage with the tone analyzer $(=0)$

2. INTUITIVENESS. The participant engaged with the tone analyzer in an intuitive way $(=1)$. The participant did not find the engagement intuitive $(=0)$.

3. UTILITY . The participant perceived the engagement with the tone analyzer as useful $(=1)$. The participant did not see any value in the engagement $(=0)$.

The results encoded by the two authors were triangulated and discrepancies resolved by reviewing the video footage. Results are shown in Table 3.

Of the 9 participants who completed the task, all were able to successfully engage with the tone analyzer.

\section{[opens tone analyzer] Analytical... I like this.}

The 11 people who did not complete the task were not able to find the tone analyzer button to open it $(n=4)$, or opened the tone analyzer but could not access it as it popped up out of view of the Validately recorder $(n=7)$.

Most of the participants (89\%) found the tone analyzer intuitive to use:

[explores tone analyzer in detail] Oh, ok. So the brighter ones are the tones that I have used... so this shows passion, this shows spontaneity, this shows trustworthiness... I guess it's because I'm being very honest. That's cool. So I guess a good article would have a mixture of different things.

So the tone analyzer [proceeds to explore]? oh, ok! So let's see? I see what this is for. So right now it's here, it's Boring. OK. So how do I make it exciting? [writes more stuff] Ooh! It went to passionate. OK, so it's like it's analyzing my whole thing [writing] and... that's actually a really great idea. I really like the idea of it. I mean, you did not say what it was for, or explain what it was for which is fine, it's... for the user to find out what this is for, which is great.

One of the participants explored the tone analyzer, but could not understand its purpose:

$$
\begin{aligned}
& \text { The tone analyzer didn't really impact my wri- } \\
& \text { ting. }
\end{aligned}
$$

In the above example, note that the words articulated by the participant mask the underlying truth of their actions.

The usefulness of the tone analyzer was found to be high (78\%). Most participants were able to make the connection between the meaning of the tones, and how these could be used to improve their writing:

[opens tone analyzer] Blunt. I really like this... [explores]... and that's the tone I want to be able to take. I like how this tells me about what the tone of 
Table 3. Results from analysis of completed user tests $(n=9)$

\begin{tabular}{|c|c|c|}
\hline Engagement & Intuitiveness & Utility \\
\hline $100 \%$ & $89 \%$ & $78 \%$ \\
\hline
\end{tabular}

my writing is, and what the readers of my blog will think. I think that's very useful.

The tone analyzer is the coolest feature in the editor. It's helpful to students when writing an essay.

So, it says Rigorous. . . at least it's not Boring or Angry, because that's not what I was going for...

A number of participants commented on the value they perceived from the actionable insights that were added in the redesign:

\section{Conclusions}

Despite the major advances in information technology that we have witnessed over the last several decades, the task of writing remains as difficult as it was one hundred years ago. In this paper, we presented two implementations of widely available AI technologies inside widely deployed commercial Webbased text editors (TinyMCE and Textbox.io). Our user testing indicated that it was possible to create a more effective writing partnership by making the writing task easier for authors.

We evaluated the impacts of a linguistic concept/entity extraction service (IBM Watson) extended with information from a semantic knowledge graph. The components were tested using unmoderated observational techniques.

Our results showed a high perceived value in the utility of the component across insert interactions (85\%), but highlighted that this was directly related to the execution of the user interface elements. In our research, participants found the basic interactions (link insertion, panel insertion and media insertion) intuitive and useful. The concept filtering user interface element, on the other hand, was found to be unintuitive and not useful. Our findings suggest that there is a strong link between the design of the UI elements and the utility perceived by an end user.

User tests related to concept extraction were deployed online using Validately. We found that this was an efficient platform for the deployment of unmoderated user tests. Our experience also highlighted the intolerance of this testing medium to mistakes or ambiguities in the design of the test task. We observed interesting nuances in the behaviour of participants, and propose further investigation into this as a topic for future work.

We explored the role that design plays in closing the usability gap in writing systems. This study presented an early exploration into the improvement of content creation through the integration of auxiliary services into WYSIWYG editors.

We designed and implemented two user interfaces to integrate tone analysis information (using a separate IBM Watson service) into the same Web-based text editors. We demonstrated that presentation of all available technical information was unhelpful for users, but that a human-centered user interface design could result in increasing the perceived utility to $78 \%$.
Information technology is used today by over 5 billion people, an increase due primarily to the rapid spread of mobile devices [20]. Given the diversity of these consumers, it is imperative that many areas of technology initially developed for technologists be redesigned for general use. We certainly found this to be true with the tone analyzer project: without changing the underlying service, we improved usability of the interaction from almost zero to $78 \%$. The success of this outcome was squarely attributable to the change we made to integrate design-led thinking into our standard development process. We thus conclude that any addition of AI componentry into general-purpose information technology be accompanied by user-centric design and testing.

\section{Further Work}

We believe that there is both commercial and academic value in further work investigating the integration of auxiliary components into WYSIWYG editors.

Several areas of additional research would be necessary before such approaches could be generally applied in WYSIWYG editors, including looking at techniques to simplify writing for multi-lingual authors. Although it would be straightforward to add multilingual data to the knowledge graph using existing techniques, Watson's tone analysis service did not support languages beyond English at the time of our research.

The size and complexity of the knowledge graph could be grown beyond general encyclopaedic content in order to present supplementary material in progressively more contextual ways. Including data that is specific to particular industries, such as manufacturing or pharmaceutical content, and even allow for extension by customers to allow querying of companyspecific data, would be useful extensions with presumably positive benefit. It is unknown whether such extensions would necessitate additional user interaction designs. It would be unreasonable to presume that new interactions would not benefit from additional user interaction design and evaluation.

While addressing issues arising for users, we also stumbled into an unexpected quagmire of issues when introducing human designers into software developer teams. Organizational psychology research may need to be extended, or discovered and integrated into software development methodologies to resolve and avoid these problems.

We did not explore the impact on writing speed or cognitive load related to context switching while writing. Further study is warranted to establish whether writing output is enhanced by the reduction of context switching.

Finally, our experiences raise interesting questions for the IT education community. Can IT educators and program coordinators afford to exclude design from traditional technology curricula if we are to produce industry-ready IT graduates? Do our programs consider how to reduce the friction between design- 
led and technology-led approaches to creating digital products? We contend that this is an important topic that warrants input from a wide range of perspectives across several disciplines.

\section{Acknowledgements}

The authors would like to thank James Johnson for prototyping IBM Watson integration into Ephox editors, Sebastian Cascajares and Mark Terry for prototyping the tone analyzer, George Wilson for his review, and Ephox Corporation for supporting this work. We would also like to thank IBM for providing us with access to the Watson services.

\section{REFERENCES}

[1] Ariely, D. Predictably irrational, HarperCollins, 278-9, 2008.

[2] Auer, S., Bizer, C., Kobilarov, G., Lehmann, J., Cyganiak, R., \& Ives, Z. Dbpedia: A nucleus for a web of open data, The semantic web, 722-735, 2007.

[3] Betella, A., \& Verschure, P. F. The affective slider: a digital self-assessment scale for the measurement of human emotions, PloS one, 11(2), e0148037, 2016.

[4] Bollacker, K., Evans, C., Paritosh, P., Sturge, T., \& Taylor, J. Freebase: a collaboratively created graph database for structuring human knowledge, Proc. 2008 ACM SIGMOD international conference on management of data, AcM, 12471250, June 2008.

[5] Cyganiak, R., Wood, D., \& Lanthaler, M. RDF 1.1 concepts and abstract syntax, W3c Recommendation, 25(02), 2014.

[6] Denzin, N. K., \& Lincoln, Y. S. (eds). The Sage handbook of qualitative research, Sage, 2011.

[7] Derkert, P., Höök, K., Fernaeus, Y., Sundström, P., Ståhl, A., Cele, G., Bresin, R., Sjölinder, M., Svensson, M., Bylund, M. \& Olausson, P. Emotions through Graphics Depicting Biometrical Sensor Data, 2006.

[8] De Weck, O. L., Roos, D., \& Magee, C. L. Engineering systems: Meeting human needs in a complex technological world, MIT Press, 2011.

[9] Ferrucci, D., Brown, E., Chu-Carroll, J., Fan, J., Gondek, D., Kalyanpur, A. A., Lally, A., Murdock, J.W., Nyberg, E., Prager, J. \& Schlaefer, N. Building Watson: An overview of the DeepQA project, AI magazine, 31(3), 59-79, 2010.

[10] Harris, S., Seaborne, A., \& Prud'hommeaux, E. SPARQL 1.1 query language, W3C recommendation, 21 March 2013,
Online available from https://www.w3.org/TR/sparql11-query/

[11] McGrenere, J., Baecker, R. M., \& Booth, K. S. An evaluation of a multiple interface design solution for bloated software, Proc. SIGCHI conference on Human factors in computing systems, ACM, 164-170, April 2002.

[12] Miles, A., \& Bechhofer, S. SKOS simple knowledge organization system reference, W3C Recommendation, 18 August 2009, Online available from https://www.w3.org/TR/skos-reference/

[13] Nielsen, J. R.i.p. wysiwyg, October 2005. Online available from https://www.nngroup.com/articles/rip-wysiwyg/.

[14] Nijdam, N. A. Mapping emotion to color, Human Media Interaction, 2-9, 2009.

[15] Ou, L. C., Luo, M. R., Woodcock, A., \& Wright, A. A study of colour emotion and colour preference. Part III: Colour preference modeling. Color Research \& Application, 29(5), 381-389, 2004.

[16] Park, W. K. Mobile phone addiction In Mobile Communications, 253-272, 2005.

[17] Prud'hommeaux, E., \& Buil-Aranda, C. SPARQL 1.1 federated query, W3C Recommendation, 21 March 2013, Online available from https://www.w3.org/TR/sparql11-federated-query/

[18] Ranjan, R. Explore in docs, sheets and slides makes work a breeze - and makes you look good, too, September 2016, Online available from https://www.blog.google/products/docs/exploredocs-sheets-and-slides/.

[19] Singhal, A. Introducing the knowledge graph: things, not strings, May 2012, Online available from https://googleblog.blogspot.com.au/2012/05/introducingknowledge-graph-things-not.html.

[20] Statista. Number of mobile phone users worldwide from 2013 to 2019 (in billions), August 2017, Online available from https://www.statista.com/statistics/274774/forecast-ofmobile-phone-users-worldwide/.

[21] Suchanek, F. M., Kasneci, G., \& Weikum, G. Yago: A large ontology from wikipedia and wordnet, Web Semantics: Science, Services and Agents on the World Wide Web, 6(3), 203-217, 2008

[22] Wallace, D. F. Transcription of the 2005 Kenyon Commencement Address, May 21, 2005, Online available from http://moreintelligentlife.com/story/david-foster-wallace-inhis-own-words. 